\title{
DISCLAIMER:
}

This document does not meet the current format guidelines of the Graduate School at The University of Texas at Austin.

It has been published for informational use only. 
Copyright

by

Emmanouil Karantinos

2015 
The Report Committee for Emmanouil Karantinos

Certifies that this is the approved version of the following report:

\section{A General Method for the Selection of an Optimum Choke Management Strategy}

\section{APPROVED BY \\ SUPERVISING COMMITTEE:}

Supervisor:

Mukul M. Sharma

Larry W. Lake 


\title{
A General Method for the Selection of an Optimum Choke Management Strategy
}

by

\section{Emmanouil Karantinos, B.A.}

\author{
Report \\ Presented to the Faculty of the GraduateSchool of \\ The University of Texas at Austin \\ in Partial Fulfillment \\ of the Requirements \\ for the Degree of
}

Master of Science in Engineering

The University of Texas at Austin

December 2015 


\section{Dedication}

To my family and friends. 


\section{Acknowledgements}

First and foremost, I would like to thank my advisor, Dr. Mukul M. Sharma for his guidance and mentorship throughout the tenure of this project. A great advisor and mentor, always eager to share knowledge and expertise transformed this two-year journey into an inspiring experience and acted as a catalyst for the continuation of my studies towards a $\mathrm{PhD}$.

I would also like to thank PGE professors for providing students with an outstanding educational experience and particularly Dr. Larry Lake for teaching us the Art of numerical simulation.

My gratitude to the member companies of the JIP Hydraulic Fracturing and Sand Control and especially Schlumberger for providing financial support for many projects,

including this one. Special thanks go to Joseph Ayoub and Mehmet Parlar for their and technical contribution to the project.

Finally, I would like to thank my family and friends for their unwavering support and love throughout the entire duration of my graduate studies. 


\title{
Abstract \\ A General Method for the Selection of an Optimum Choke Management Strategy 1
}

\author{
Emmanouil Karantinos, MSE \\ The University of Texas at Austin, 2015
}

Supervisor: Mukul M. Sharma

Different choke management strategies have been adopted by operators in the field. However, no general method exists for systematically selecting an optimum choke management strategy. In this study, we propose a general method for the selection of bean-up duration and bean-up strategy (choke management), that will maximize well productivity by minimizing formation damage, reducing sand production and reducing the impact of multiphase flow effects.

Strategies of stepwise bottom-hole pressure (BHP) adjustments are compared using the principle of superposition and the analytical solution of the diffusivity equation for vertical wells operating under different choke settings. The optimum choke management strategy is based on the anticipated formation damage mechanisms. For

\footnotetext{
${ }^{1}$ The context of this report has also been published at the SPE European Formation Damage Conference and Exhibition: "E. Karantinos, M. M. Sharma, J. Ayoub, M. Parlar, R. Chanpura, A General Method for the Selection of an Optimum Choke Management Strategy, paper SPE 174196 presented at the SPE European Formation Damage Conference and Exhibition, Budapest, 03-05 June 2015" J. Ayoub, M. Parlar and R. Chanpura provided guidance and technical expertise.
} 
example, if fines migration and sand production are a concern, then minimizing the near wellbore pressure gradients is the primary criterion for the selection of the optimum choke management strategy.

Results indicate that the selection of the optimum strategy (sequence of choke settings) is independent of the drawdown and depends primarily on the duration of the ramp-up period. Among the 251 strategies evaluated, three of them consistently appear to be the optimum. We further refine strategies using a single parameter, incorporated in a dimensionless expression of BHP as a function of time. From simulation results, we conclude that for relatively short bean-up durations (i.e., infinite acting behavior), the pressure gradient reduction at the wellbore varies logarithmically with time. Choke management strategies appear to have similar performance as far as no more than $70 \%$ of the drawdown is applied during the initial $20 \%$ of the overall bean-up duration. For longer durations (i.e., when the presence of the no-flow boundary is felt), the optimum strategy depends on both reservoir properties and radial extent. For the case of square drainage area, a plot suggesting the optimum strategy with respect to dimensionless duration is presented. Positive skin and multiphase flow near the wellbore, negatively affect the performance of bean-up strategies. For vertical wells producing from multiple layers, bean-up strategies should be selected with respect to the effective horizontal permeability, ultimately yielding the greatest pressure gradient reduction in the low permeability zones.

The proposed method enables engineers to select the optimum choke management strategy with respect to bean-up duration and formation properties. The duration of the 
bean-up process is drawdown dependent thus further research is encouraged in determining the maximum allowable pressure gradient in order to curtail sand production. 


\section{Table of Contents}

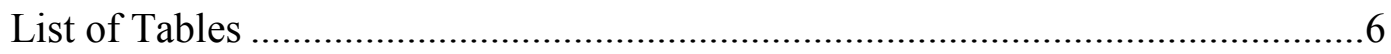

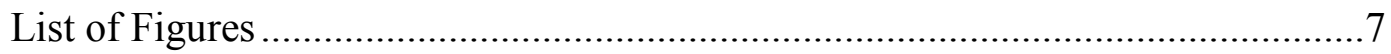

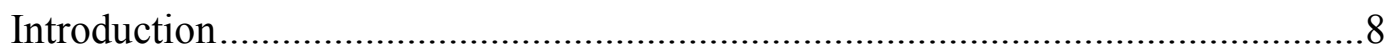

Choke Management Considerations ............................................................. 10

A general framework for comparing bean-up operations ............................... 12

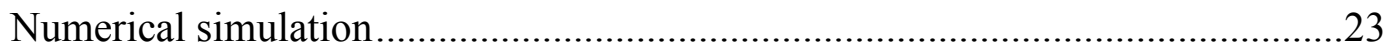

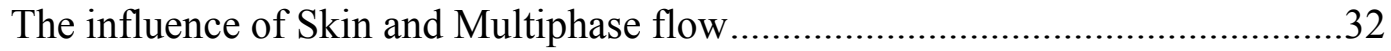

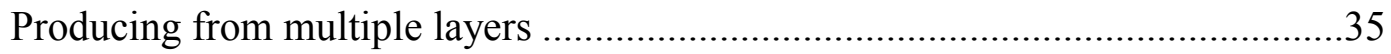

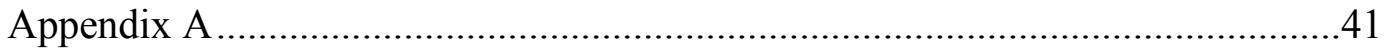

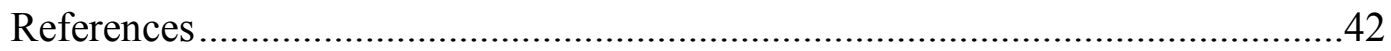




\section{List of Tables}

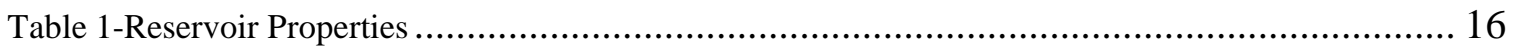

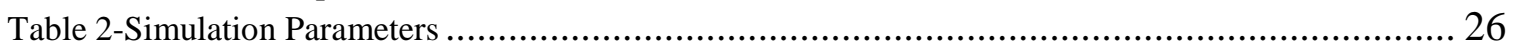




\section{List of Figures}

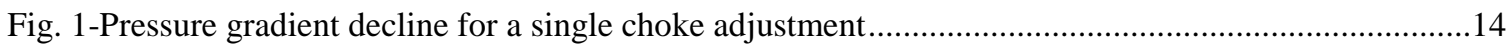

Fig. 2-Lattice discretization of BHP and bean-up duration. ....................................................................

Fig. 3-Variation of pressure gradients (dashed lines) for the applied bean-up (continuous line). The dotted

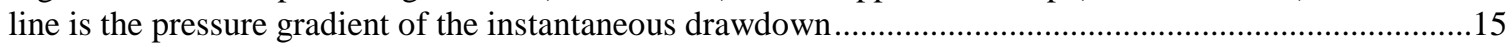

Fig. 4-Comparing choke management strategies with respect to pressure gradient reduction and cumulative

production for a bean-up duration of $10 \mathrm{hr}$. Reservoir properties shown in Table 1..................................17

Fig. 5-Parameters $\lambda$ and RCP for all 251 strategies. Bean-up duration is $10 \mathrm{hr}$ and the reservoir properties

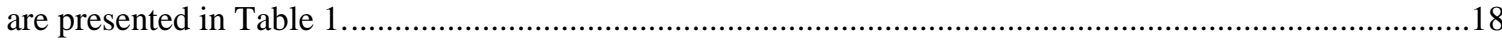

Fig. 6-Choke management strategies yielding the minimum pressure gradients.........................................19

Fig. 7-Ratio of pressure gradient versus bean-up duration for the three optimum strategies. Reservoir

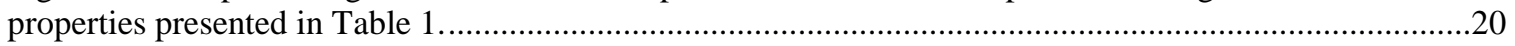

Fig. 8-Dimensionless graph of BHP and bean-up time for different values of parameter $a \ldots \ldots \ldots \ldots \ldots \ldots \ldots \ldots \ldots . . .24$

Fig. 9-Bean-up operation defined from Eq.(6) for $a=-0.9$ and choke settings of 15 min each. ...................24

Fig. 10-Pressure gradient ratio versus bean-up duration for the case of an oil well in a 1,400x1,400 ft square

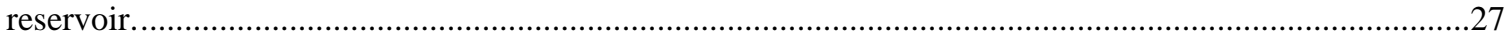

Fig. 11-Comparing choke management strategies for an oil well producing from a square reservoir of

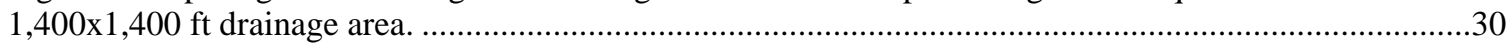

Fig. 12-Comparing choke management strategies for a gas well producing from a square reservoir of

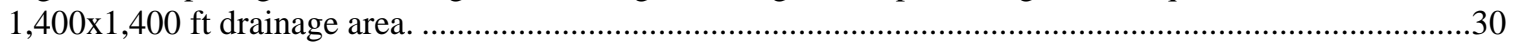

Fig. 13-Optimum bean-up strategy versus relative bean-up duration for homogeneous and isotropic

reservoirs of square drainage area. ................................................................................................

Fig. 14-Ratio of pressure gradients versus bean-up duration for different values of S. $k=1 \mathrm{md}, 1,400 \times 1,400$

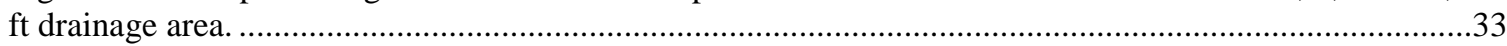




\section{Introduction}

Choke management strategies vary significantly among operators, primarily with respect to the overall duration of the ramp up process. An improper production schedule, characterized by a rapid and excessive drawdown could trigger massive sand production or proppant flowback, possibly resulting in completion impairment and wellbore failure.

Previous studies on bean-up protocols and sand production (Weingarten \& Perkins 1995; Tiffin et al. 2003; Wong et al. 2003) have focused primarily on suggesting the maximum allowable drawdown or upper bound limits for fluid velocities in the near wellbore region, with respect to different failure mechanisms and completion types. Such recommendations are either based on compilation of data or have been derived from conventional models of tensile and/or shear failure. The application of analytical models usually provides an overly conservative estimate for the maximum allowable drawdown since sand production is considered to be concomitant with sand failure. Additional parameters affecting sanding severity include reservoir depletion and water breakthrough, which significantly reduces tensile strength. Researchers have underlined the notion that sand failure is a necessary; however not a sufficient condition for sand production to occur. Sanding events will only be triggered upon the presence of favorable hydraulic conditions (i.e., substantial pressure gradients) capable of mobilizing the failed sand or causing direct tensile failure of the weakly consolidated formation (Vaziri et al. 2002).

Based on the previous observation, choke management strategies should be aimed towards minimizing the near-wellbore pressure gradients induced by the applied overall drawdown. This notion has also been adopted by Geilikman et al. (2005) who studied the effect of bean-up procedures on fines migration. Keeping these potential formation 
damage mechanisms in mind we have proposed a method to select a bean-up or choke management strategy that minimizes the pressure gradient in the near wellbore region. The approach is quite general and can be applied whether the well is unloaded for the first time, pushed toward a peak rate or re-started after a long shut-in period. Within this study we assess the performance of different choke management strategies for wells that operate under constant BHP, rather than increasing surface rate. More specifically, we provide a framework for selecting an optimum series of decreasing BHP that will yield the greatest reduction in pressure gradients in the well vicinity, thus minimizing the risk of formation tensile failure and/or sand fluidization. The method is general and could potentially be applied in cased and perforated wells where screen erosion or gravel pack destabilization are the dominant failure mechanisms. In the later case, the optimization process should focus on minimizing pressure gradients or fluid velocities along perforations. For open-hole completions, strategies are compared using the available analytical solution for wells operating under constant BHP whereas, for a more detailed analysis, numerical simulation is performed. Additionally, we address how the overall drawdown and bean-up duration may affect the selection of the optimum choke management strategy as well as the potential benefits of prolonging the overall duration of the ramp-up process. Finally, we assess the performance of bean-up strategies in vertical wells characterized by positive or negative skin factors as well as for vertical wells producing from multiple layers. 


\section{Choke Management Considerations}

Choke management strategies (or bean-up operations) refer to the process of gradually increasing the rate or drawdown towards achieving a target, stabilized rate. A bean-up operation can be described with respect to increasing production rates or levels of drawdown. However, since the rate is primarily controlled by the choke size and since most of the previous work has focused on determining the maximum allowable drawdown, we adopt the latter approach. In order to simplify the study of bean-up operations, we assume that the wellbore provides adequate vertical lift performance to produce all the fluid the reservoir can deliver during the ramp-up process. Consequently, bean-up operations can be studied on the basis of increasing levels of drawdown or, equivalently, decreasing BHP. Taking the previous considerations into account, a beanup process can be defined by the following parameters: duration $\left(t_{\mathrm{B}}\right)$, overall drawdown (DD), number of BHP configurations $(\mathrm{N})$, duration and magnitude of each subsequent reduction in BHP. Even though the term bean-up operation usually refers to the process of bringing a well on production, choke management strategies should also be considered during shut-in cycles. In fact, frequent and harsh shut-downs followed by rapid bean-ups can severely harm cementation due to differential strain loading, causing premature formation failure and possibly sanding (Vaziri et al. 2004).

Among the different parameters characterizing a bean-up operation, drawdown has been studied the most. Several papers have been published for predicting the maximum allowable drawdown that a formation or completion can withstand

(Nouri et al. 2006; Wong et al. 2003). Methods vary from purely empirical to analytical models and sophisticated numerical schemes. However, since the parameters associated with numerical modeling are not routinely measured, major operating companies 
typically deploy analytical models that are based on either shear or tensile failure criteria. Such analytical models typically capture a single failure mechanism and assume that formation or completion failure is concomitant with the onset of sand production. Vaziri et al. (2002) showed that analytical models generally provide a high level of conservatism in predicting the maximum allowable drawdown, especially in HP/HT wells. Additionally, massive reservoir depletion and/or water-breakthrough limit the applicability and reliability of analytical methods (Nouri et al. 2006).

As opposed to the maximum drawdown, bean-up duration is a parameter that has not been extensively studied. Vaziri et al. (2004) suggests that a new step of the ramp-up process should not be applied unless pore pressures from the previous adjustment have reached a state of equilibrium within a close region around the well. That implies that formations characterized by high diffusivity can be brought on production within relatively short bean-up durations (Geilikman et al. 2005). Later in this study we address how the duration of the ramp-up process can determine the selection of the optimum choke management strategy with respect to reservoir and fluid properties. 


\section{A general framework for comparing bean-up operations}

In this section we present the foundation for comparing choke management strategies in reservoirs that satisfy the assumptions of the radial diffusivity equation. Bean-up operations will be evaluated with respect to minimizing pressure gradients near the wellbore. Even though the assumptions associated with the radial diffusion equation impose limitations in the applicability of the method in real-life reservoirs, useful observations and general conclusions may be drawn.

For a single choke adjustment (or reduction in BHP), the pressure solution can be obtained from the analytical solution of the transient radial diffusion equation for wells produced under constant BHP. The well is located in the center of a circular reservoir and satisfies the assumptions inherent in the radial diffusivity equation: the formation is considered to be homogeneous and isotropic with constant thickness, porosity and permeability. Additionally, the pore space is occupied by hydrocarbon of constant viscosity which is assumed to be slightly compressible. A brief review of the analytical solution is presented in Appendix A and a thorough overview is provided by Economides (1979). The dimensionless pressure or rate decline solution is given in tabulated form as well as in the Laplace space. Rather than using tabulated forms, MathWorks MATLAB was deployed in order to apply the principle of superposition and numerically invert Laplace transforms, ultimately obtaining the solution into real time and space variables.

Fig. 1 illustrates the rate (or pressure gradient decline) as a function of dimensionless time, $\mathrm{tD}$, for a single reduction in BHP. The dimensionless time is defined as:

$$
\mathrm{t}_{\mathrm{D}}=\frac{\mathrm{kt}}{\varphi \mu \mathrm{cr}_{\mathrm{w}}^{2}}
$$


The pressure gradient obtains its maximum value immediately after a decrease in BHP. The rate of decline depends on the diffusivity constant, D, which depends on both formation and fluid properties:

$$
\mathrm{D}=\frac{\mathrm{k}}{\varphi \mu \mathrm{c}}
$$

A reservoir with high diffusivity will result in a rapid decline in pressure away from the wellbore upon the implementation of a single, instantaneous drawdown.

Bean-up operations are intended to reduce the wellbore flowing pressure from an initial pressure $\left(\mathrm{P}_{i}\right)$ to a final pressure $\left(\mathrm{P}_{f}\right)$ over the duration of the rate ramp-up process. Pressure, $\mathrm{P}_{i}$, can either be the initial reservoir pressure or the average pressure after a sufficiently long shut-in period. The difference between $\mathrm{P}_{i}$ and $\mathrm{P}_{f}$ equates the overall drawdown (DD) of the ramp-up process, which is user specified. In order to systematically define choke management strategies, we provide the discretization shown in Fig.2. The BHP is considered to be constant during every step of the ramp-up process. Different strategies can be selected, allowing for a reduction of BHP in a stepwise manner. Enumeration of the alternate strategies (paths) using a repetition scheme yields a total of 252 strategies, including the instantaneous drawdown case. 


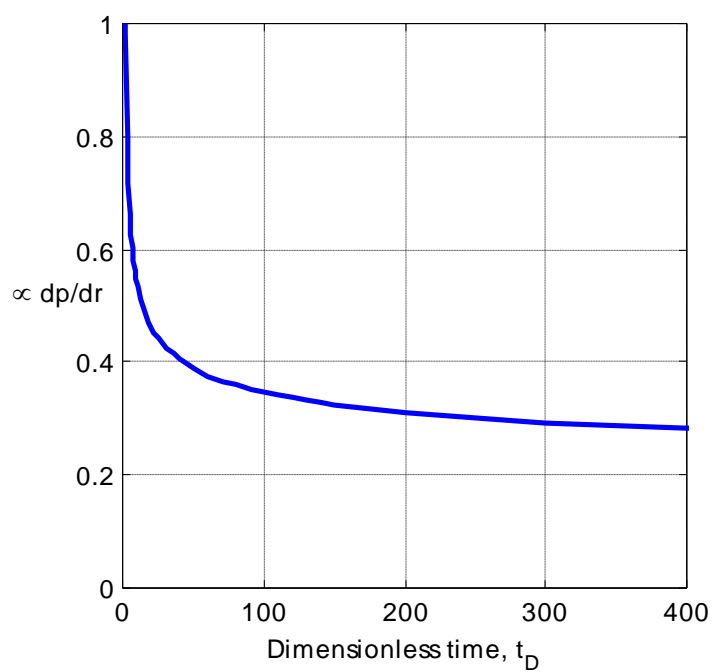

Fig. 1-Pressure gradient decline for a single choke adjustment

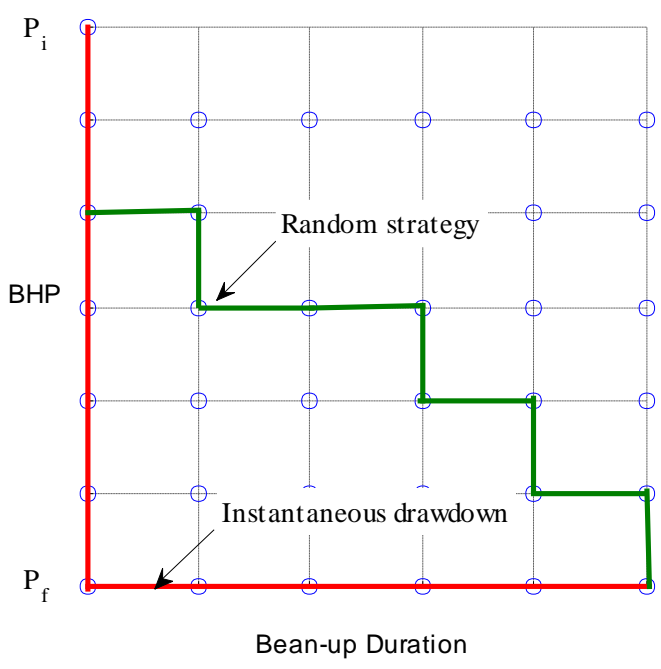

Fig. 2-Lattice discretization of BHP and bean-up duration.

For a stepwise reduction in BHP, the pressure solution can be obtained by applying the principle of superposition with respect to the time variable. Several examples of the principle of superposition for wells producing under constant pressure are presented by Lee (1982). The principle of superposition allows us to calculate the pore pressure gradient near the wellbore after each choke adjustment. Pressure gradients are calculated using the superimposed rates and Darcy's equation at the wellbore diameter. More specifically, the pressure gradient is calculated at the sandface 90 seconds after a choke adjustment, for bean-up operations as well as for the instantaneous drawdown case. Prior to the passage of 90 seconds, the pressure gradients will be greater compared to the calculated values however, the time frame of 90 seconds is assumed to be representative of the maximum pressure gradient during a given choke adjustment and short enough to inhibit severe formation damage or sanding events of continuous nature. The selection of 90 seconds is rather arbitrary, and affects neither the process of comparing choke management strategies nor the optimum strategy itself. 
Fig.3 illustrates the pressure gradient induced by a random bean-up operation. The reservoir properties are presented in Table 1. The BHP is gradually reduced until an overall drawdown of 1,200 psi is applied over a period of $10 \mathrm{hr}$. Fig. 3 indicates that the maximum pressure gradient during the ramp-up process is smaller (by approximately $18 \%$ ) compared to the maximum pressure gradient induced by a single, instantaneous reduction in the BHP by 1,200 psi. Gradually reducing the BHP has a profound impact on pressure gradients near the wellbore.

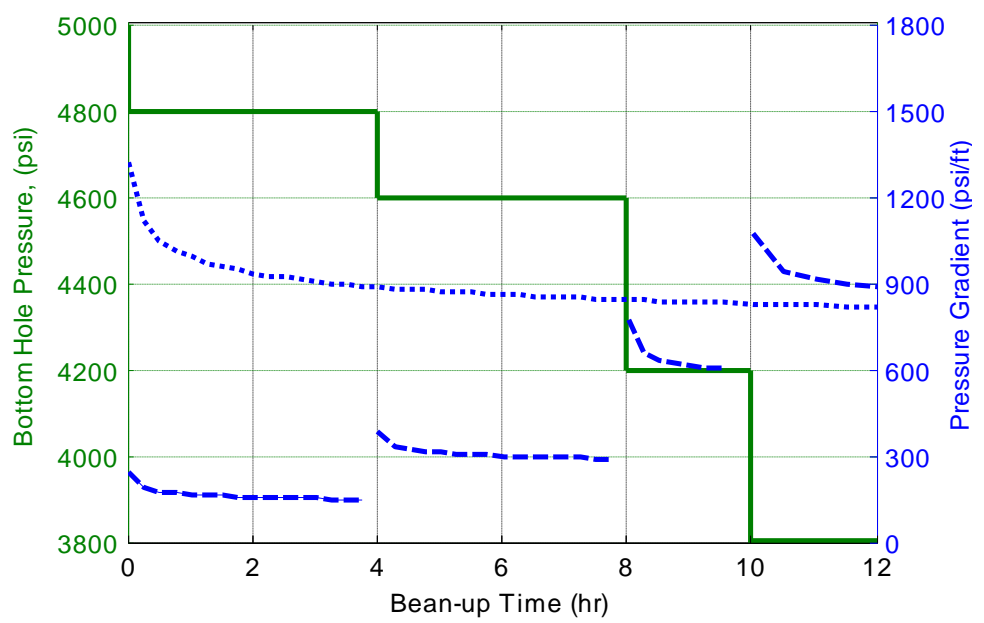

Fig. 3-Variation of pressure gradients (dashed lines) for the applied bean-up (continuous line). The dotted line is the pressure gradient of the instantaneous drawdown 
Table 1-Reservoir Properties

\begin{tabular}{ll}
\hline Reservoir Property & Value \\
\hline Permeability, $\mathrm{k}(\mathrm{mD})$ & 100 \\
Porosity, $\varphi$ & 0.2 \\
Total compressibility, c (psi-1) & $5 \times 10-5$ \\
Fluid viscosity, $\mu(\mathrm{cP})$ & 1 \\
Drainage radius, re (ft) & 500 \\
Wellbore radius, rw (ft) & 0.25 \\
\hline
\end{tabular}

In order to quantify the efficiency of a bean-up operation, we define the following two parameters:

A) $\lambda$ is the ratio of the maximum pressure gradient during the entire ramp-up process over the (maximum) pressure gradient of the instantaneous drawdown:

$$
\lambda=\frac{(\mathrm{dp} / \mathrm{dr})_{\text {bean-up }}}{(\mathrm{dp} / \mathrm{dr})_{\text {inst. drawdown }}}
$$

B) RCP (Ratio of Cumulative Production) is the ratio of the cumulative volume produced during the ramp-up process over the volume produced during the corresponding instantaneous drawdown for the same bean-up duration:

$$
\mathrm{RCP}=\frac{(\text { Volume Produced })_{\text {bean-up }}}{(\text { Volume Produced })_{\text {inst. drawdown }}}
$$

Volumes are calculated by adding the cumulative production of individual solutions, based on the principle of superposition.

Resulting from the definition, both parameters are smaller than one. We also need to reiterate that the smaller the parameter $\lambda$, the greater the efficiency of the ramp-up process with regard to pressure gradient reduction. A parametric analysis with respect to the applied drawdown proves that both ratios are independent of the overall drawdown. 
That implies that the efficiency of a strategy is not related to the magnitude of the drawdown.

It is important to note that the optimum strategy is not known a priori. Fig.4 illustrates three different strategies along with the corresponding parameters, $\lambda$ and RCP. Intuition suggests that a stepwise strategy characterized by a linear-like reduction in BHP would be a good option (Fig. 4a). However, an aggressive reduction in BHP during the early stage of the ramp-up process (Fig. $\mathbf{4 b}$ ) performs better, yielding lower pressure gradients (smaller value of parameter $\lambda$ ). On the contrary, poor performance is observed when a considerable reduction in BHP is applied toward the final stage of a bean-up operation (Fig. 4c). The reservoir response to the aforementioned strategies can be explained as follows: applying a substantial drawdown during the early stage of the beanup allows pressure gradients to dissipate with time, resulting in smaller gradients as additional drawdown is applied towards the final stage of the ramp-up process. In other words, a large reduction in BHP has a smaller contribution to the pressure gradient if applied during the early stage of a bean-up operation.

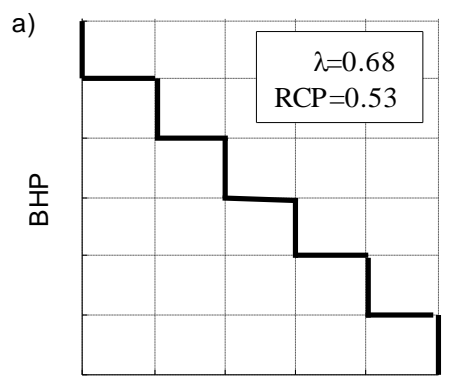

Bean-up Time

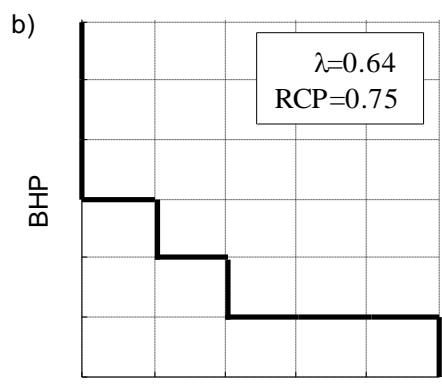

Bean-up Time

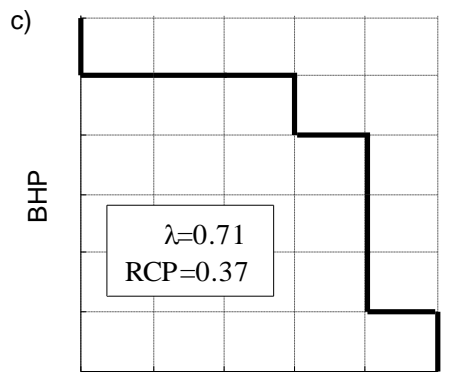

Bean-up Time

Fig. 4-Comparing choke management strategies with respect to pressure gradient reduction and cumulative production for a bean-up duration of $10 \mathrm{hr}$. Reservoir properties shown in Table 1.

Fig. 5 presents parameters $\lambda$ and RCP for the entire set of 251 strategies (i.e. 252 strategies less the instantaneous drawdown case), for a bean-up duration of $10 \mathrm{hr}$. Every 
point on this graph represents a different bean-up operation. Apart from minimizing pressure gradients near the wellbore, achieving a relatively high cumulative production during the ramp-up process could also be an additional objective, particularly in high-rate wells if a prolonged bean-up operation is applied. The graph illustrates that selecting a bean-up process from the upper-left part of the graph can accomplish both objectives (low pressure gradients and high cumulative production). Thus, low pressure gradients and high cumulative production are not mutually exclusive. On the other hand, selecting a bean-up strategy from the bottom-right part of the plot is expected to underperform, yielding high pressure gradients and small volumes of cumulative production. Such inefficient strategies are characterized by a significant reduction in BHP toward the $\mathrm{mid} /$ final stage of the ramp-up process (see Fig. 3).

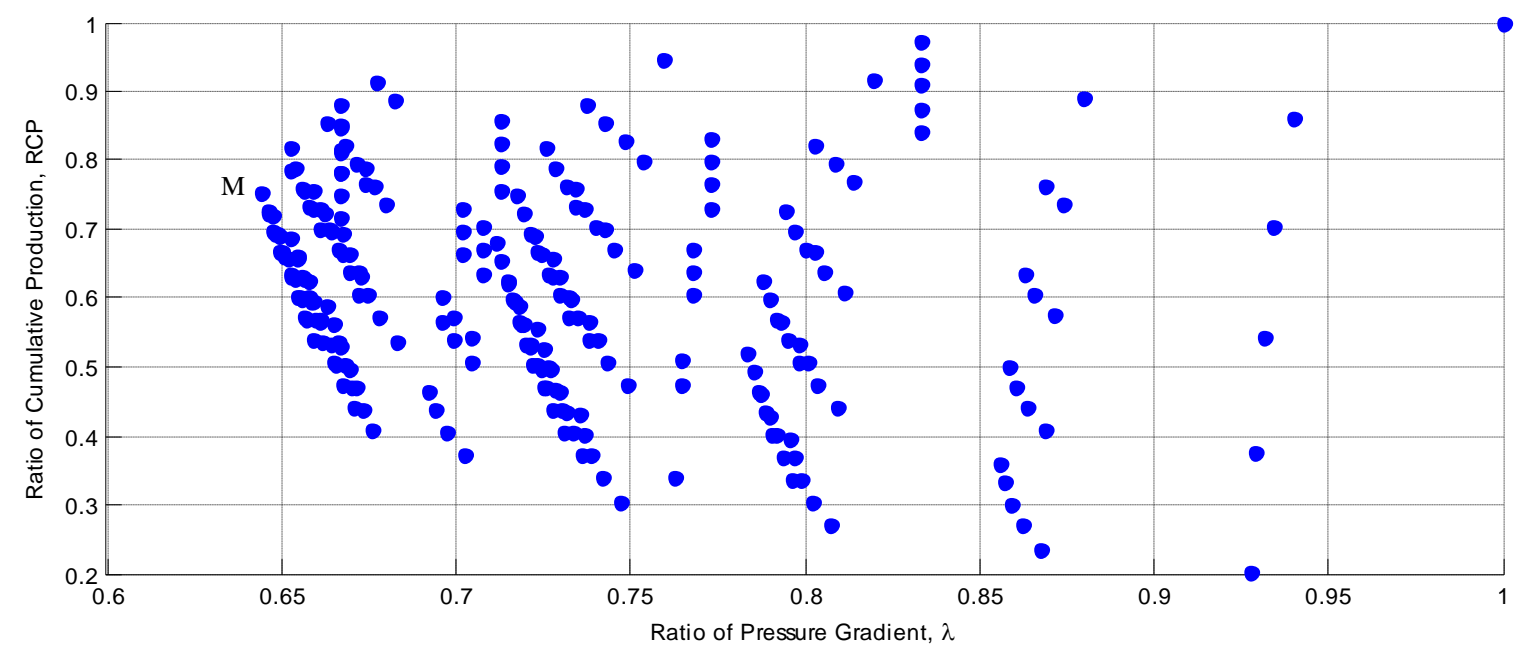

Fig. 5-Parameters $\lambda$ and RCP for all 251 strategies. Bean-up duration is $10 \mathrm{hr}$ and the reservoir properties are presented in Table 1.

Nevertheless, minimizing pressure gradients during the bean-up operation is the primary objective of this study. To this end, we need to identify the strategy that yields the minimum value of $\lambda$. This strategy is considered to be the optimum choke management strategy. For example, in Fig. 5, the optimum strategy corresponds to point 
M. To identify the optimum strategies, multiple comparisons were performed for a wide range of the following parameters: diffusivity constant, reservoir radial extent and beanup duration. The runs indicate that among the 251 strategies, three of them consistently appear to be the optimum. The optimum strategies are presented in Fig.6. It is important to note that all three optimum strategies fall below the dashed diagonal.
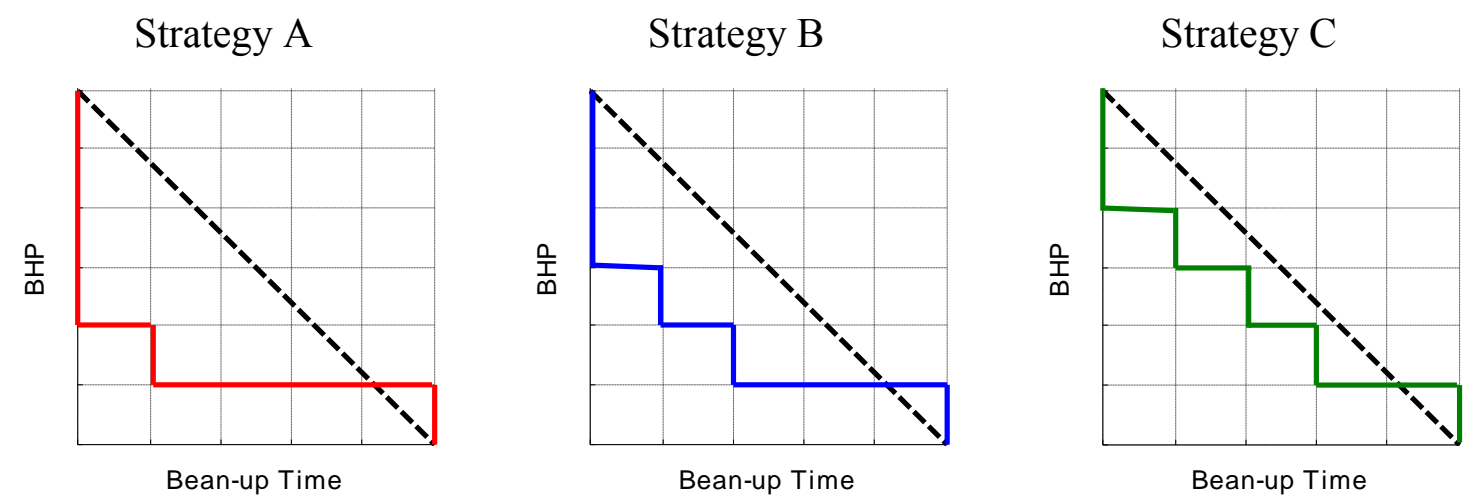

Fig. 6-Choke management strategies yielding the minimum pressure gradients.

The performance of strategies $\mathrm{A}, \mathrm{B}$ and $\mathrm{C}$ for various bean-up durations is presented in Fig.7. Any strategy other than A, B or C lies above the curves shown. Also, the vertical dashed line indicates the end of infinite acting period, $t_{E I A}$, calculated by the formula proposed by Earlougher (1977), in field units:

$$
\mathrm{t}_{\mathrm{EIA}}(\mathrm{hr})=1200 \frac{\varphi \mu \mathrm{c}}{\mathrm{k}} \mathrm{r}_{\mathrm{e}}^{2}
$$




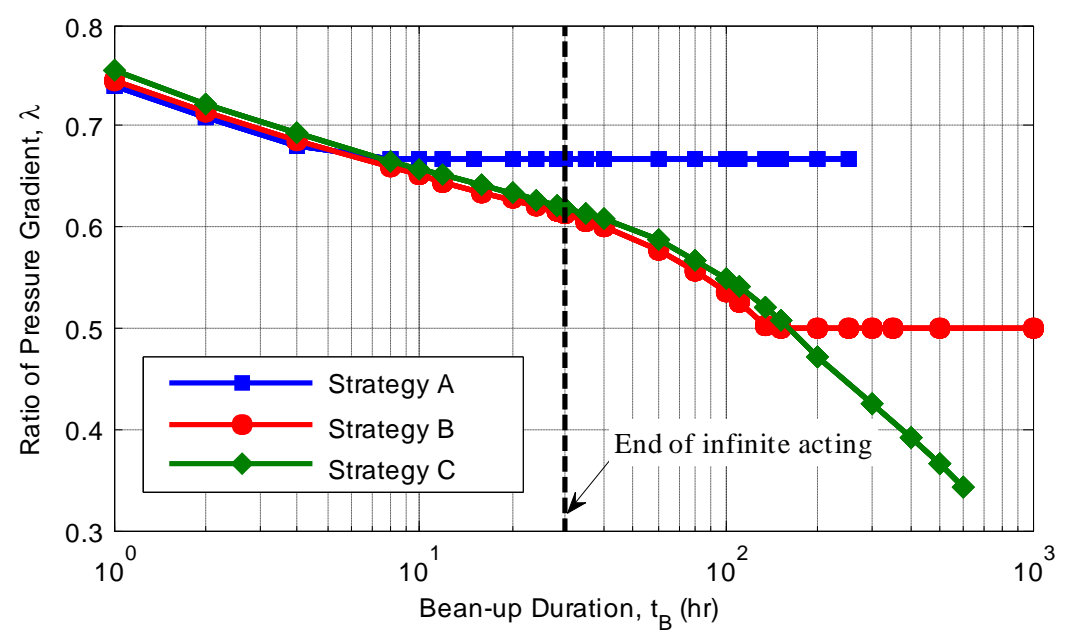

Fig. 7-Ratio of pressure gradient versus bean-up duration for the three optimum strategies. Reservoir properties presented in Table 1.

We observe that for a short bean-up, lasting less than $5 \mathrm{hr}$, all three strategies have similar performance, with Strategy A performing slightly better. In this case, the fastest way to bring a well on production corresponds to strategy A. For bean-up durations greater than a critical value (here equal to 8 hours) the maximum pressure gradient of Strategy A will always occur during the initial stage of the process, regardless of the duration of the ramp-up operation. The initial reduction in BHP proves to be the critical factor of the process, locking the performance of Strategy A at 0.67. This justifies the plateau observed (see Fig. 6) and constitutes a limitation of the proposed discretization. For bean-up operations longer than $5 \mathrm{hr}$ but shorter than the tEIA, strategies $\mathrm{B}$ and $\mathrm{C}$ have similar performance. For prolonged bean-up operations strategy $\mathrm{C}$ is the optimum. We also observe that prolonging the duration of Strategy $\mathrm{C}$ further reduces pressure gradients: for Strategy $\mathrm{C}$, the maximum pressure gradient is always observed towards the final stage of the process and the initial reduction in BHP is not a limiting factor. This justifies the fact that no plateau is observed for strategy C. In general, the following trend is observed: increasing the bean-up duration causes the optimum bean-up strategy to 
shift towards a less aggressive reduction in BHP (gradually progressing from Strategy A to $\mathrm{C})$.

Fig. 7 also indicates that $\lambda$, which is proportional to the maximum pressure gradient, decreases logarithmically with bean-up duration, provided that the optimum choke strategy is selected. For durations lasting longer than $t_{\mathrm{EIA}}$, pressure gradients rapidly decline, as a result of reservoir depletion. If reservoir depletion occurs before the end of the bean-up process, rate decline causes additional decrease in pressure gradients, which justifies the deviation from the original slope.

In this section, we performed a comparison of 251 bean-up operations derived from a lattice discretization. The discretization is rather coarse allowing for a maximum of six choke adjustments, with an initial reduction in BHP at the beginning of the process. Since we only used a maximum of six choke adjustments, this may pose limitations in the applicability of the method for prolonged bean-up operations. However, with the current analysis, the following conclusions can be drawn:

- The performance of bean-up operations is independent of the overall applied drawdown. Consequently, determining the maximum allowable drawdown and selecting a choke management strategy are two distinct tasks that should be conducted independently and iteratively to yield the optimum short-term production schedule.

- Low pressure gradients and relatively high volumes of cumulative production are not mutually exclusive, provided that an optimum strategy 
is selected. In general, a better performance is observed if a large reduction in BHP is applied during the early stage of the ramp-up process.

- The performance of a choke management strategy depends on the duration of the bean-up process as well as on reservoir properties. For relative short durations (i.e., infinite acting behavior), a large initial reduction in BHP is preferred (Fig. 6a) whereas, for a prolonged bean-up operation a more gradual adjustment is suggested (Fig. 6c).

- For relatively short bean-up operations (i.e., infinite acting behavior), pressure gradients reduce logarithmically with increasing duration. The impact of reservoir boundaries increases the performance bean-up operations due to reservoir depletion. Thus, prolonging the duration of the ramp-up process could prove beneficial in reservoirs characterized by high diffusivity. 


\section{Numerical simulation}

The method described in the previous section is general and can be used for comparing choke management strategies in any reservoir of interest, provided that the transient pressure solution is known. For cases where the strict assumptions of the analytical solution are not satisfied, a reservoir simulator can be deployed to acquire the pressure solution and the corresponding pore pressure gradients near the wellbore. This section describes the process of comparing bean-up operations in oil and gas wells using a reservoir simulator.

As mentioned previously, the objective is to minimize pressure gradients near the wellbore. A reservoir simulator provides the capability of calculating the pressure gradients at any point of interest, depending on the anticipated failure mechanism. For a slotted liner or open hole completion, pressure gradients may be calculated at the sandface or within the plastic zone. For a cased and perforated well, emphasis should be placed on minimizing pressure gradients along the perforations in order to prevent screen erosion and/or destabilization of the gravel pack. The near-wellbore region should be meshed accordingly in a refined manner. Data including spatially varying formation properties, phase behavior, reservoir shape and well location can be in incorporated within the input file of the reservoir simulator in use. This work was performed with Computer Modeling Group (CMG) IMEX, a black-oil commercially available reservoir simulator.

In order to overcome the shortcomings of the previously presented coarse discretization of the choke settings, we introduce the following single-parameter dimensionless equation that describes the variation of BHP as a function of time: 


$$
\frac{\mathrm{P}_{\mathrm{wf}}\left(\mathrm{t}_{\mathrm{BD}}\right)-\mathrm{P}_{\mathrm{f}}}{\mathrm{P}_{\mathrm{i}}-\mathrm{P}_{\mathrm{f}}}=\frac{(\mathrm{a}+1)\left(1-\mathrm{t}_{\mathrm{BD}}\right)}{\mathrm{a}\left(1-\mathrm{t}_{\mathrm{BD}}\right)+1},
$$

where $t_{\mathrm{BD}}$ is the dimensionless bean-up time, defined as

$$
\mathrm{t}_{\mathrm{BD}}=\frac{\mathrm{t}}{\mathrm{t}_{\mathrm{B}}} \leq 1
$$

Eq. (6) was derived on the premise that the implemented BHP is somewhat inversely proportional to time. The equation was adjusted so that at $\mathrm{tBD}=0$ and $\mathrm{tBD}=1$ the BHP equals the initial and final pressure of the bean-up process. The left-hand side of Eq.(6) is the fraction of the cumulative drawdown applied at time, $t_{\mathrm{BD}}$, of the ramp-up process. Parameter, $a$, represents different choke management strategies, as shown in Fig. 8. The instantaneous drawdown case corresponds to $a=-1$, whereas for $a=0$, the BHP is linearly reduced with time. For positive values of $a$, the BHP configuration is located in the upper-right triangular section of Fig. 8. As discussed previously, the optimum BHP configuration falls under the diagonal, thus, from now on we will focus exclusively on negative values of $a$.

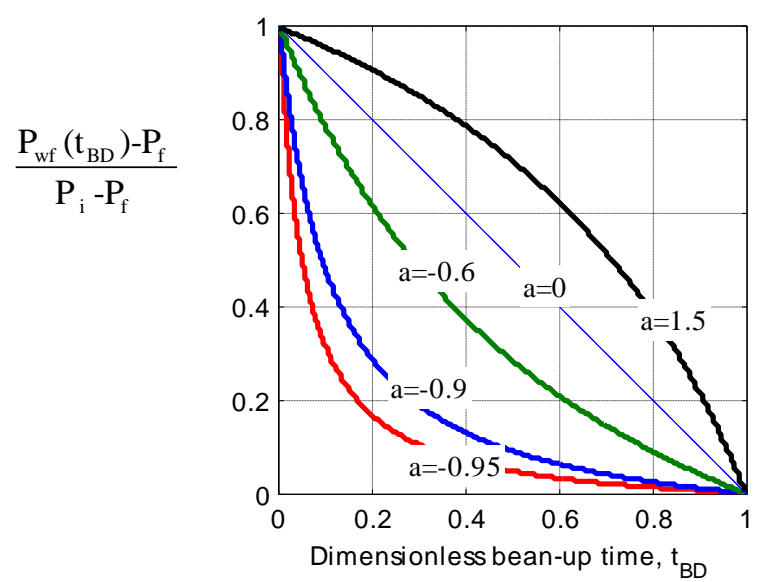

Fig. 8-Dimensionless graph of BHP and bean-up time for different values of parameter $a$

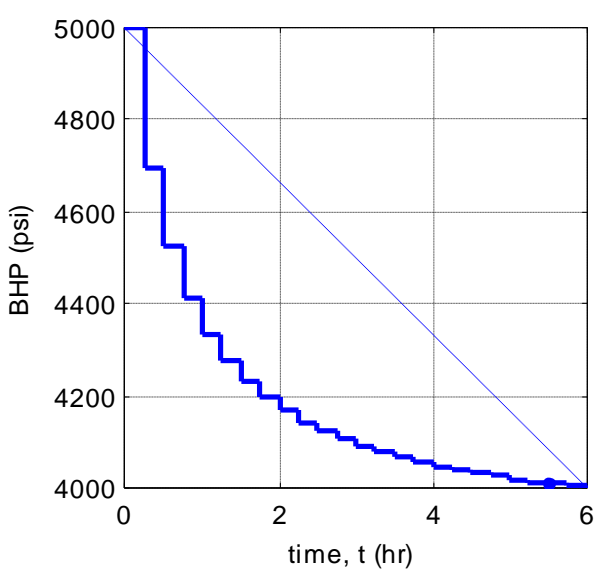

Fig. 9-Bean-up operation defined from Eq.(6) for $a=-$ 0.9 and choke settings of 15 min each. 
In order to simulate and ultimately compare choke management strategies, the continuously varying wellbore pressure, as calculated by Eq. (6) is converted into steps of constant BHP. The duration of each step is considered to be equal to $15 \mathrm{~min}$. Fig. 9 presents a BHP schedule derived in this manner. The proposed steps of constant BHP can be implemented in the field with the use of a computer-adjusted choke. 
Different bean-up scenarios (values of $a$ ) are compared for oil and gas wells located at the center of a homogeneous and isotropic square reservoir. The reservoir and bean-up properties are presented in Table 2. The combination of reservoir properties and bean-up parameters yields a pool of possible scenarios. Pressure gradients are calculated $0.2 \mathrm{ft}$ away from the wellbore using a refined mesh of square elements $(0.2 \times 0.2 \mathrm{ft})$ in the well vicinity. The wellbore comprises of cells operating under constant BHP (i.e., infinite productivity index).

Table 2-Simulation Parameters

\begin{tabular}{ll}
\hline Formation Properties & \\
\hline Field dimensions (ft x ft) & $600 \mathrm{x} 600 ; 1,400 \mathrm{x} 1,400$ \\
Porosity, $\varphi$ & 0.15 \\
Permeability, $k(\mathrm{mD})$ & $1 ; 10 ; 100 ; 1000$ \\
Temperature, $\mathrm{T}(\mathrm{F})$ & 250 \\
Initial Reservoir Pressure, $\mathrm{P}_{i}(\mathrm{psi})$ & 5,000 \\
Irreducible water saturation, $\mathrm{S} w r$ & 0.25 \\
Rel. permeability exponent, $n$ & 2.5 \\
\hline Bean-up Properties & \\
\hline Total drawdown (psi) & 1,$000 ; 2,000 ; 3,200$ \\
Duration, $t_{\mathrm{B}}$ (hr) & $2 ; 4 ; 8 ; 12 ; 24 ; 48$ \\
Bean-up strategy, $a$ & -0.99 to $24(20$ values) \\
\hline Oil Reservoir - Fluid Properties & \\
\hline Oil density (API) & 30 \\
Specific gravity of gas (air $=1)$ & 0.70 \\
Viscosity, $\mu$ (cP) & $\sim 0.75$ \\
Compressibility (psi-1) & $\sim 3 \times 10^{-5}$ \\
Reference Pressure Pref (psi) & 3500 \\
Reference Temperature Tref (F) & 250 \\
Bubble point pressure, Pb(psi) & 3,000 \\
Residual oil saturation, Sor & 0.25 \\
\hline Gas Reservoir - Fluid Properties & \\
\hline Specific gravity of gas (air $=1)$ & 0.70 \\
Viscosity, $\mu$ (cP) & $\sim 0.025$ \\
Reference Pressure Pref (psi) & 3500 \\
Reference Temperature Tref (F) & 250 \\
Residual gas saturation, Sgr & 0.15 \\
\hline
\end{tabular}


For each set of formation and fluid properties, choke management strategies (parameter $a$ ) are evaluated for bean-up durations $\left(t_{B}\right)$ varying from 2 to $48 \mathrm{hr}$. For each $t_{B}$, the optimum strategy is selected. Fig. 10 illustrates the reduction in pressure gradients with increasing bean-up duration for different values of formation permeability in an oil well producing from a $1,400 \times 1,400 \mathrm{ft}$ reservoir. For durations shorter than $t_{E I A}$ (i.e., infinite acting behavior) pressure gradients reduce logarithmically with $t_{B}$. On the other hand, if the no-flow boundary is felt during the bean-up process, greater pressure gradient reduction can be achieved. This observation implies that bean-up operations are expected to be more efficient in fields characterized by high diffusivity, where $t_{E I A}$ is limited to a couple of hours. Eq. (2) suggests that the fluid component of the diffusivity constant is the product of viscosity and compressibility which is greater by approximately an order of magnitude for the case of a gas hydrocarbon. Consequently, the effect of bean-up operations will be more pronounced in high permeability, gas fields.

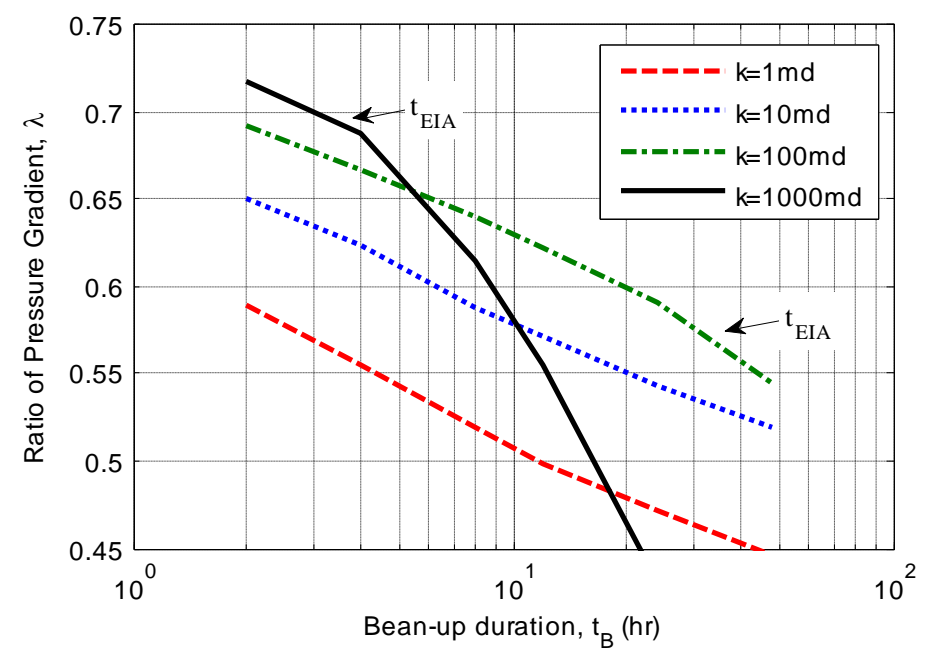

Fig. 10-Pressure gradient ratio versus bean-up duration for the case of an oil well in a 1,400x1,400 ft square reservoir. 
For each set of formation and fluid properties, choke management strategies (parameter $a$ ) are evaluated for bean-up durations $\left(t_{B}\right)$ varying from 2 to $48 \mathrm{hr}$. For each $t_{B}$, the optimum strategy is selected. Fig. 10 illustrates the reduction in pressure gradients with increasing bean-up duration for different values of formation permeability in an oil well producing from a $1,400 \times 1,400 \mathrm{ft}$ reservoir. For durations shorter than $t_{E I A}$ (i.e., infinite acting behavior) pressure gradients reduce logarithmically with $t_{B}$. On the other hand, if the no-flow boundary is felt during the bean-up process, greater pressure gradient reduction can be achieved. This observation implies that bean-up operations are expected to be more efficient in fields characterized by high diffusivity, where $t_{E I A}$ is limited to a couple of hours. Eq. (2) suggests that the fluid component of the diffusivity constant is the product of viscosity and compressibility which is greater by approximately an order of magnitude for the case of a gas hydrocarbon. Consequently, the effect of bean-up operations will be more pronounced in high permeability, gas fields.

Figs. 11 and 12 present the performance (parameter $\lambda$, y-axis) of different choke settings (parameter $a$, x-axis) with respect to permeability and bean-up duration for oil and gas wells. Based on these plots, the following observations can be made:

- For infinite acting behavior (see Figs. 11a, 11b, 11c, 12a, 12b), strategies between $a=-0.9$ to $a=0$ have similar performance. In fact a BHP setting with $a=-0.9$ performs slightly better. This observation is in agreement with the conclusions derived from the discretized model discussed in the previous section.

- For relatively short bean-up operations (i.e., infinite acting behavior), the best way to bring a well on production, even for a bean-up duration of 2 
hr, corresponds to a parameter of -0.9. Fig. 8 suggests that such a strategy can be implemented in the field by applying no more than $70 \%$ of the overall drawdown during the initial $20 \%$ of the bean-up duration. On the contrary, values of parameter $a$ smaller than -0.95 yield higher pressure gradients and should be avoided.

- For the case when reservoir boundary effects can be felt (see Figs. 11d, 12c, 12d) the optimum choke strategy shifts from $a=-0.9$ to greater values, depending on the duration of the ramp-up process. More precisely, the longer the bean-up duration, the larger the value of parameter $a$. 

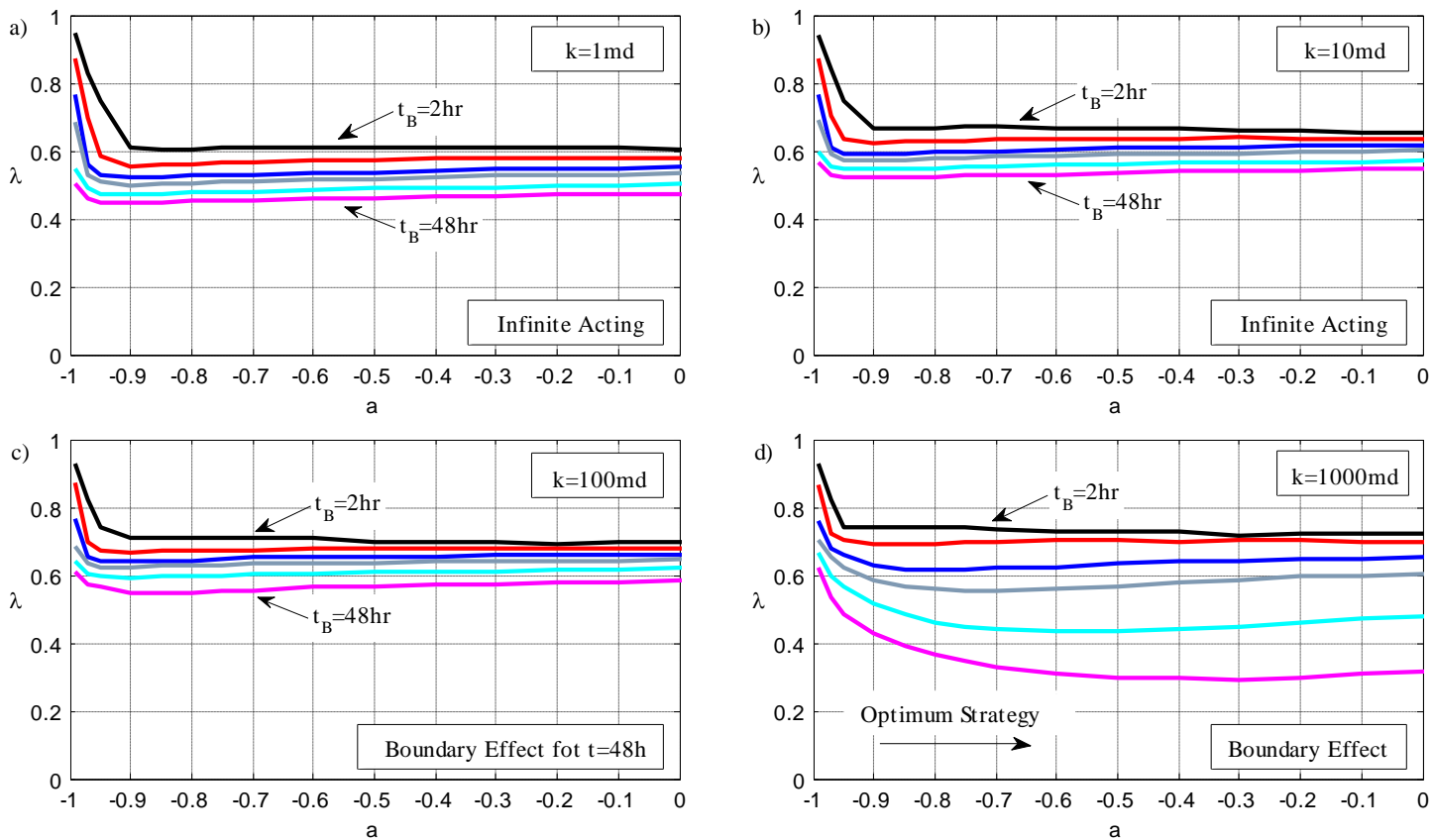

Fig. 11-Comparing choke management strategies for an oil well producing from a square reservoir of $1,400 \times 1,400 \mathrm{ft}$ drainage area.
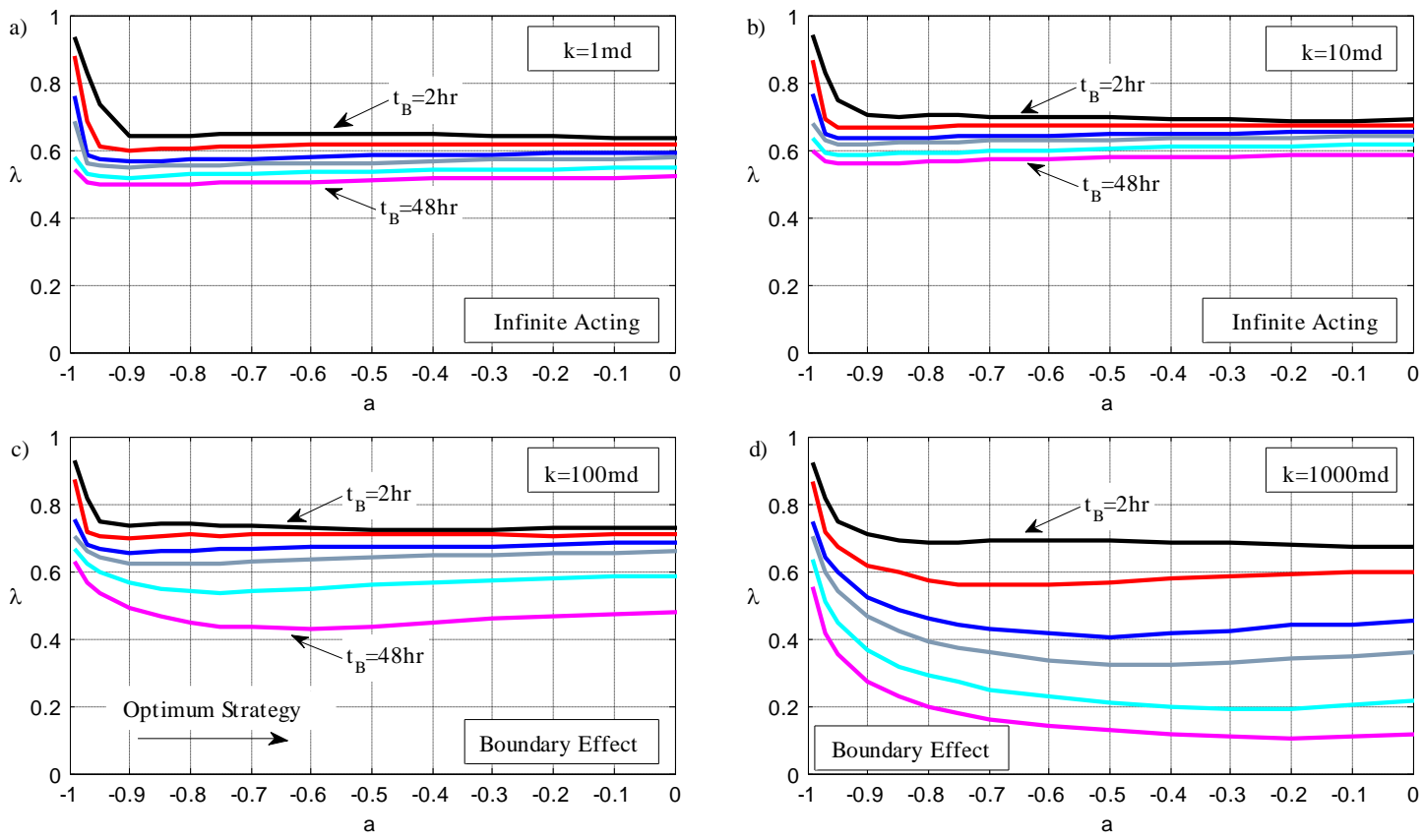

Fig. 12-Comparing choke management strategies for a gas well producing from a square reservoir of $1,400 \times 1,400 \mathrm{ft}$ drainage area. 
Simulation results were utilized to express the optimum strategy as a function of bean-up duration, formation and fluid properties. To this end, we define the relative duration $t^{*}$ :

$$
\mathrm{t}^{*}=\frac{\mathrm{t}_{\mathrm{B}}}{\mathrm{t}_{\mathrm{EIA}}},
$$

which provides a relative measure of the bean-up duration with respect to reservoir properties and acreage. For a homogeneous and isotropic reservoir with square drainage area, the optimum strategy can be obtained from Fig. 13. The points have been generated using Eq. (5) and approximate values from Table 2 for both oil and gas wells. The increasing trend between duration and parameter $a$ indicates that a prolonged bean-up operation should be combined with a slower reduction in BHP.

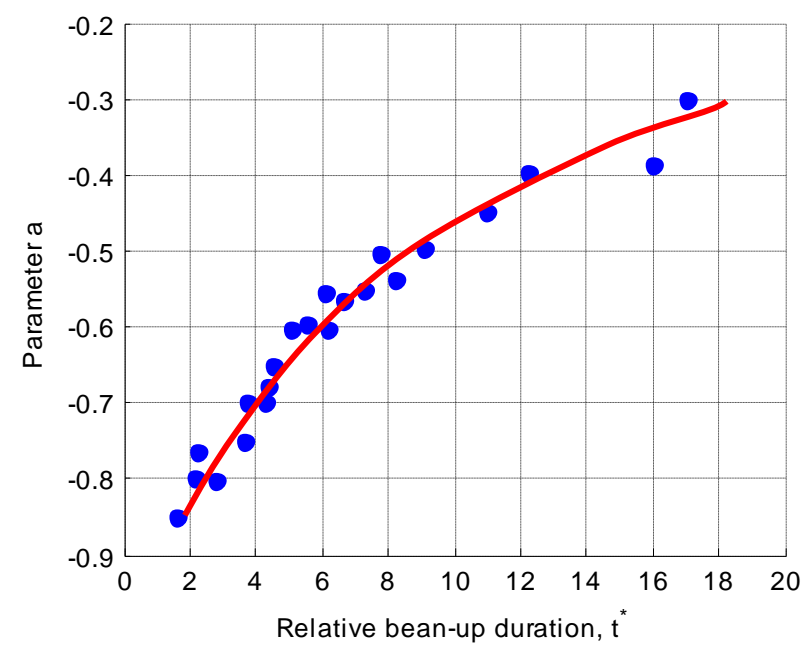

Fig. 13-Optimum bean-up strategy versus relative bean-up duration for homogeneous and isotropic reservoirs of square drainage area. 


\section{The influence of Skin and Multiphase flow}

Permeability near the wellbore may be impaired due to plugging of the pore space by formation fines, inefficient filter cake removal or considerable increase in effective stress. Engineers may account for these factors with a positive skin factor however, in the current model, near wellbore permeability is assumed to be constant and indifferent to alterations in effective stress or cavities formed due to sand production. In this section we assess the effect of skin and multiphase flow on the performance of choke management strategies. More specifically we investigate whether such conditions can boost or compromise the efficiency of bean-up operations with respect to reducing pressure gradients near the wellbore.

A positive or negative skin factor can be implemented in the model by assuming an impaired or stimulated zone of constant permeability $k_{\text {skin }}$ near the wellbore. The permeability of the impaired (or stimulated) zone can be obtained from the following equation:

$$
S=\left[\frac{k}{k_{\text {skin }}}-1\right] \ln \left[\frac{r_{\text {skin }}}{r_{w}}\right]
$$

Using the reservoir properties of Table 2, the near-wellbore permeability was modified to account for an impaired zone of $3 \mathrm{ft}$. The methodology of the previous section is adopted with pressure gradients calculated within the impaired zone for the instantaneous drawdown case as well as for each candidate bean-up operation. Fig. 14 illustrates the reduction in pressure gradients with respect to increasing bean-up duration for different values of skin. Positive values of skin negatively affect the performance of bean-up operations, yielding higher values of $\lambda$ and thus larger pressure gradients, 
relative to the corresponding instantaneous drawdown. On the contrary, the presence of a stimulated zone increases diffusivity near the well, ultimately improving the performance of bean-up operations. We also observe that prolonging the duration of a ramp-up process has a more profound effect on a stimulated well. For $\mathrm{S}=-2$, increasing the bean-up duration from 4 to $8 \mathrm{hr}$ further reduces $\lambda$ by $10 \%$ (from point $\mathrm{A}$ to point $\mathrm{B}$ ) whereas for $S=3$ pressure gradients only reduce by an additional $4 \%$ (point $C$ to point $D$ ).

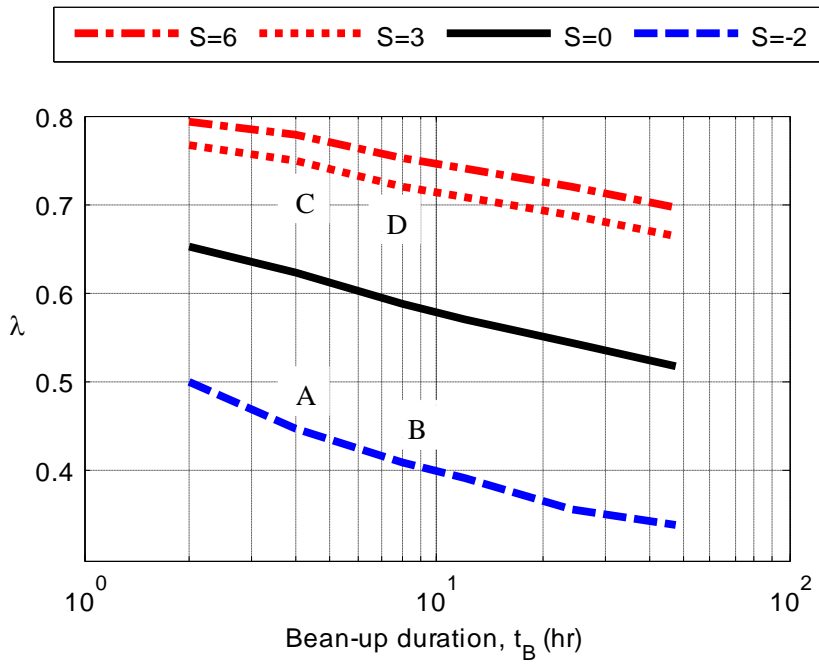

Fig. 14-Ratio of pressure gradients versus bean-up duration for different values of $\mathrm{S}$. $k=1 \mathrm{md}, 1,400 \times 1,400 \mathrm{ft}$ drainage area.

Multiphase flow near the wellbore may occur due to various reasons including water coning, flow of condensate or BHP dropping below the bubble point. Multiphase flow near the wellbore gives rise to relative permeability, which reduces the effective permeability. The reduction in effective permeability is equivalent to the presence of a positive skin factor, with the implications presented previously. Choke management strategies were compared for wellbore pressures dropping below the bubble point and the results indicate an effect similar to that of a positive skin factor. The impact of multiphase 
flow appears to depend on the endpoint values as well as on the shape of the relative permeability curves. 


\section{Producing from multiple layers}

So far, we have focused exclusively on vertical wells producing from a single horizontal layer. However, most wells are completed along multiple layers or produced from a combination of normally pressured and over pressured productive zones. In this section, we discuss in a qualitative manner how the presence of multiple producing layers may affect the selection of an optimum bean-up strategy.

Fig. 15 illustrates a well intersecting two layers of different permeabilities. Prior to initiating the ramp-up process, the pressure is hydrostatic. The vertical permeability of the formation is assumed to be spatially constant and equal to $1 \mathrm{md}$. Additionally, the BHP is considered to be constant along the vertical portion of the well since a wellbore model has not been incorporated.

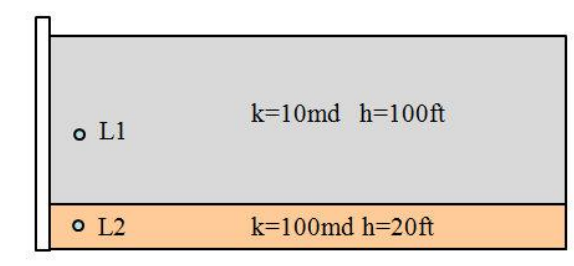

Fig. 15-Simple model of vertical gas well producing from two layers of different permeabilities.

The well is subjected to an instantaneous drawdown. The abrupt nature of the applied drawdown, combined with low vertical permeability disrupts the hydrostatic pressure distribution near the wellbore. At the very early stage of production, both layers behave autonomously, as if the presence of the other is neglected. That causes pressure gradients to be different among the layers, giving rise to higher pressure gradients in the low permeability zone (point L1). However, with increasing time, vertical pressure equilibrium is restored and pressure gradients converge to an equal value. 
Using a bean-up operation allows vertical pressure equilibrium to re-establish shortly after the first few choke adjustments. In this case, pore pressure gradients are approximately equal in both layers and the formation behaves like a medium with horizontal permeability equal to:

$$
\mathrm{k}_{\mathrm{av}}=\frac{\mathrm{h}_{1} \mathrm{k}_{1}+\mathrm{h}_{2} \mathrm{k}_{2}}{\mathrm{~h}_{1}+\mathrm{h}_{2}}
$$

Fig. 16 presents the performance of different choke management strategies applied on a gas well producing from both layers I and II. Bean-up operations are compared for a duration of $48 \mathrm{hr}$ in a gas reservoir with drainage area of 1,400x1,400 ft. The graph includes the performance of different choke management strategies for the sequence of layers as well as for the case where each layer is produced independently. From this graph, we may infer the following:

- The optimum choke management strategy for a well producing from both layers corresponds to a value of $a$ equal to -0.85 (Points A and B). This strategy is different compared to the optimum strategy of $a=-0.6$ which should have been applied if Layer II was to be produced independently (Point C). Consequently, the optimum strategy for a well producing from a series of layers should not be approximated by using individual layer permeabilities, but rather the weighted average horizontal permeability.

- The coupling of both layers yields greater pressure gradients (Point A) in the high permeability layer compared to the anticipated pressure gradient if that layer was the only one to be produced (Point C). On the other hand, smaller pressure gradients are observed in the low permeability layer. 
Consequently, the coupling of layers proves to be beneficial for the low permeability layer and detrimental for the sand prone, high permeability layer. That implies that in order to limit the maximum pressure gradient under a threshold value, smaller overall drawdowns should be applied in multi-layered formations compared to fields comprising a single highpermeability layer of identical properties.

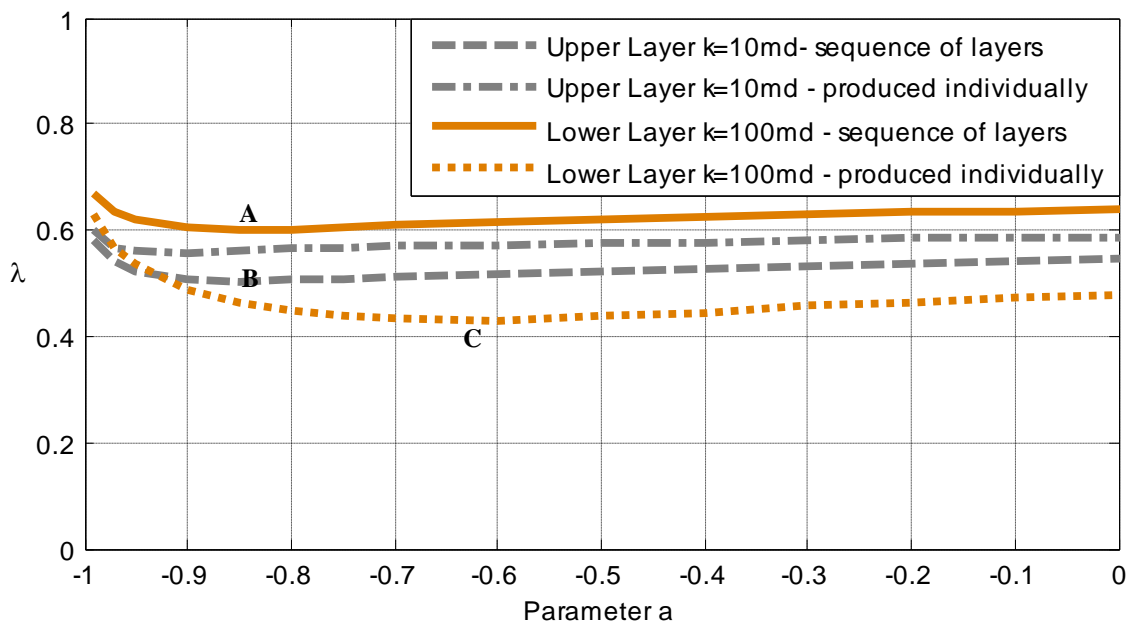

Fig. 16-Comparing choke management strategies in a gas well producing from two layers. Bean-up duration is $48 \mathrm{hr}$ and the drainage area is $1,400 \times 1,400 \mathrm{ft}$. 
In this study we provided a general framework and a systematic method for comparing and selecting choke management strategies. Examples were illustrated for vertical, openhole oil and gas wells located at the center of homogeneous and isotropic reservoirs of circular and square drainage area. The method is general and can be used in any real-life reservoir. The proposed method is intended to be used in conjunction with either analytical pressure transient models or reservoir simulators that provide an estimate of the pressure transient response of wells with varying drawdowns. The process for selecting an optimum choke management strategy can be summarized in the following steps:

\section{Step 1-Determine the maximum allowable drawdown}

a) A conservative estimate can be obtained from analytical models that account for shear and/or tensile failure. Finite element modeling is suggested in cases where reservoir depletion and water cut limit the reliability of analytical models.

b) In weakly consolidated formations, a proxy can be obtained from drawdown guidelines based on compilation of data and screen erosion criteria (Tiffin et al. 2003).

\section{Step 2-Determine the maximum allowable pressure gradient}

The maximum pressure gradient can be obtained from analytical models based on direct tensile or cavity failure. The critical pressure gradient can also be approximated through Darcy's Law from rate (or fluid velocity) limitations (Wong et al. 2003) and field specific formation properties. Laboratory experiments can provide additional verification to ensure that the selected pressure gradient will not cause massive fluidization of the disaggregated material. 


\section{Step 3-Determine the duration of the ramp-up process}

Construct a graph of $\lambda$ vs bean-up duration $\left(t_{\mathrm{B}}\right)$, similar to Fig. 10, and use this to select a bean-up duration ensuring that the pressure gradient stays below the maximum pressure gradient obtained in Step 2.

\section{Step 4-Select the optimum choke management strategy}

For the selected drawdown and bean-up duration, select the optimum choke management strategy (value of $a$ ) by constructing a plot of $\lambda$ vs $a$ for the selected bean-up duration $\left(t_{\mathrm{B}}\right)$, similar to Fig.11.

Within this study we also evaluated the effect of skin and provided a qualitative assessment of how choke management strategies should be selected in multi-layered formations. The following practical conclusions may be drawn from this study:

- For relatively short durations (i.e., infinite active behavior), bean-up strategies have similar performance as far as no more than $70 \%$ of the overall drawdown is applied within the initial $20 \%$ of the bean-up process.

This recommendation can be used as a rule of thumb to inhibit excessive pressure gradients near the wellbore.

- For longer bean-up operations (i.e., when the effect of the no-flow boundaries is felt), the BHP should be reduced more gradually. For a prolonged bean-up, the BHP should be reduced linearly with time.

- For relatively short bean-up operations (i.e., infinite acting behavior), pressure gradients reduce logarithmically with increasing bean-up duration. Boundary effects have a positive influence on the performance of bean-up operations as a result of reservoir depletion. That implies that 
in reservoirs of high diffusivity (high perm gas) we may prolong the duration to benefit from boundary effects.

- Positive skin factors and multiphase flow negatively affect the performance of bean-up operations since they limit diffusivity and thus the dissipation of pressure gradients near the wellbore.

- In multilayered formations, the optimum choke management strategy should be selected with respect to the weighted average horizontal permeability. The co-existence of layers causes pressure gradients to be relatively elevated in the high-permeability, sand prone layers which may pose additional limitations to the maximum allowable drawdown. 


\section{Appendix A}

Equation (11) constitutes the dimensionless form of the transient radial diffusion equation:

$$
\frac{\partial^{2} P_{D}}{\partial r_{D}^{2}}+\frac{1}{r_{D}} \frac{\partial P_{D}}{\partial r_{D}}=\frac{\partial P_{D}}{\partial t_{D}}
$$

For the case of a well producing from the center of a circular reservoir under constant bottom hole pressure $\left(\mathrm{P}_{\mathrm{wf}}\right)$ the dimensionless variables are defined as:

$$
\begin{gathered}
r_{D}=r / r_{w} \\
t_{D}=\frac{k t}{\varphi \mu c r_{w}^{2}} \\
P_{D}\left(r_{D}, t_{D}\right)=\frac{P_{i}-P(r, t)}{P_{i}-P_{w f}} \\
q_{D}=\frac{q \mu}{2 \pi k h\left(P_{i}-P_{w f}\right)}
\end{gathered}
$$

The dimensionless pressure solution $\mathrm{P}_{\mathrm{D}}$ and dimensionless production rate $\mathrm{q}_{\mathrm{D}}$ can be obtained by numerically calculating the following inverse Laplace transformations:

$$
\begin{aligned}
& P_{D}\left(r_{D}, t_{D}\right)=L^{-1}\left\{K_{o}\left(r_{D} \sqrt{l}\right) /\left[l\left(K_{o}(\sqrt{l})+s \sqrt{l} K_{1}(\sqrt{l})\right)\right]\right\} \\
& q_{D}\left(r_{D}, t_{D}\right)=L^{-1}\left\{K_{1}(\sqrt{l}) /\left[\sqrt{l}\left(K_{o}(\sqrt{l})+s \sqrt{l} K_{1}(\sqrt{l})\right]\right\}\right.
\end{aligned}
$$

In the above equations, $l$ represents the Laplace variable and $\mathrm{K}_{\mathrm{o}}$ and $\mathrm{K}_{1}$ are the modified Bessel functions of the second kind of order zero and one, respectively. A thorough overview on the solution of the radial diffusion equation is provided by Economides (1977). 


\section{References}

Earlougher RC. Advances is Well Test Analysis. SPE; 1977.

Economides C. Well Test Analysis for Wells Produced at a Constant Pressure. PhD Thesis. Stanford University: US, 1979.

Geilikman M, Dria D, Stewart D, Wong G. Bean-up Guidlines for Sand Control Completions, paper SPE 95870 presented at the 2005 SPE Annual Technical Conference and Exhibition, Dallas, TX, October 9-12.

Lee J. Well Testing. Vol 1. SPE Publications; 1982.

Nouri A, Vaziri H, Belhaj H, Islam R. Sand-Production Prediction: A New Set of Criteria for Modeling Based on Large-Scale Transient Experiments and Numerical Investigation,paper SPE 90273 presented at the 2004 SPE Annual Technical Conference and Exhibition, Houston, TX, September 26-29.

Ong S, Atlas B, Ramos R, Zheng Z. Sand Production Prediction in High Rate, Perforated and Open-hole Gas Wells, paper SPE 58721 presented at the 2000 SPE International Symposium on Formation Damage Control, Lafayette, LA, 23-24 February.

Tiffin D, Stein M, Wang X. Drawdown Guidlines for Sand Control Completions. paper SPE 84495 presented at the 2003 SPE Annual Technical Conference and Exhibition, Denver, CO, October 5-8.

Tronvoll J, Papamichos E, Skjaerstein A, Sanfilippo F. Sand Production in UltraWeak Sandstones: Is Sand Control Absolutely Necessary? paper SPE 39042 presented at the Latin American and Caribbean Petroleum Engineering Conference, Rio de Janeiro, Brazil, 30 August-3 September. 
Vaziri H, Xiao Y, Palmer I. Assessment of several sand prediction models with particular reference to HPHT wells, paper SPE 78235 presented at the 2002 SPE/ISRM Rock Mechanics Conference, Irving, TX, 20-23 October.

Vaziri H, Allam R, Kidd G, Bennett C, Grose T, Robison P, Malyn J. Sanding: A Rigorous Examination of the Interplay Between Drawdown, Depletion, Start-up Frequency and Water Cut, paper SPE 89795 presented at the 2004 SPE Annual Technical Conference and Exhibition, Houston, TX, 26-29 September.

Vaziri H, Barree B, Xiao Y, Palmer I, Kutas M. What is the Magic of Water in Producing Sand?, paper SPE 77683 presented at the 2002 SPE Annual Technical Conference and Exhibition, San Antonio, TX, 29 September-2 October.

Weingarten J, Perkins T. Prediction of Sand Production in Gas Wells: Methods and Gulf of Mexico Case Studies. JPT. 1995;47(07):596-600.

Wong G, Fair P, Bland K, Sherwood R. Balancing Act: Gulf of Mexico Sand Control Completions, Peak Rate Versus Risk of Sand Control Failure, paper SPE 84497 presented at the 2003 SPE Annual Technical Conference and Exhibition, Denver, CO, October 5-8. 